\title{
Evaluation of Dissolution Behavior of Paracetamol Suspensions
}

\author{
Md. Golam Azam ${ }^{1}$ and Syed Shabbir Haider ${ }^{2}$ \\ ${ }^{1}$ Department of Pharmacy, Northern University Bangladesh, 3/18, Iqbal Road, Mohammadpur, \\ Dhaka-1207, Bangladesh \\ ${ }^{2}$ Department of Pharmaceutical Technology, Faculty of Pharmacy, University of Dhaka, Dhaka-1000, \\ Bangladesh. Present address: Department of Pharmacy, North South University, Banani, \\ Dhaka-1213, Bangladesh
}

\begin{abstract}
Ten brands of commercial paracetamol suspensions were investigated for their dissolution characteristics in $0.1 \mathrm{~N} \mathrm{HCl}$ at a speed of $25 \mathrm{rpm}$ using USP dissolution test apparatus II (Paddle method). It was observed that differences exist among the rate of paracetamol dissolution from the samples, particularly at the early stages. For nine of the brands, paracetamol dissolution varied between $72 \%$ and $100 \%$ at 15 minutes. One of the samples showed a very unsatisfactory rate and extent of drug dissolution as only $39 \%$ and $52 \%$ paracetamol was released after 15 and 30 minutes respectively. The effect of two commonly used suspending agents, CMC-Na and Avicel, on the release of paracetamol was studied using four laboratory-made suspensions. Retarded drug dissolution was observed which was related to the concentration of the suspending agents. Drug release kinetics followed first order as well Higuchi models. Viscosity of the samples and initial drug release was found to be inversely correlated $\left(\mathrm{R}^{2}=0.9081\right.$ at 3 minutes) which faded away with the lapse of time.
\end{abstract}

Key words: Paracetamol suspension, in vitro dissolution, suspending agents

\section{INTRODUCTION}

In vitro dissolution testing offers a convenient and inexpensive means of predicting absorption and bioavailability differences among capsules and tablets formulations of the same drug. ${ }^{1}$ Several methods are employed for measuring the dissolution of common solid dosage forms like capsules and tablets. ${ }^{2}$ For a drug to be absorbed, the solid dosage form must first undergo disintegration, deaggregation and then dissolution of drug particles. Suspensions correspond to the post-disintegration stage of tablets and capsules and, thus, share dissolution as a ratelimiting step for absorption and bioavailability. So testing the suspensions for dissolution is as important as that of solid dosage forms. ${ }^{2,3}$

Correspondence to: Syed Shabbir Haider Tel: 880-2-9136406; Fax: 880-2-8615583

E-mail: hshabbir@bdmail.net
Several investigators showed that the relationship between dissolution and the suspension formulation is very important. ${ }^{3}$ In particular, suspending agents are of great importance in suspension dissolution. ${ }^{3-6}$ Various additives, methods of preparation (flocculating, deflocculating procedures) and aging were reported to influence the dissolution of suspensions. A number of reports revealed unsatisfactory bioavailability of drugs from suspensions due to poor dissolution behavior and in most cases, the problems were found to be linked with the suspending agents added in the products. ${ }^{1,2,10-13}$ These findings suggest the possibility of drug adjuvant interactions in suspensions formulations that could affect drug dissolution and absorption. 
After the 1990's paracetamol tragedy in Bangladesh, almost all of the local manufacturers switched over to the suspension dosage form withdrawing their solution products and presently over 100 pharmaceutical companies are marketing paracetamol suspensions in this country. ${ }^{14}$ Paracetamol, a familiar OTC analgesic and antipyretic drug, is sparingly water soluble and prone to dissolution and bioavailability problems. Suspension dosage form may further enhance this problem if not properly formulated. The objective of this research work was to study the comparative dissolution behavior of marketed paracetamol suspensions. This investigation also focused on some other pharmaceutic parameters like viscosity and suspending agent concentration which can directly or indirectly affect paracetamol dissolution.

\section{MATERIALS AND METHODS}

Materials. Paracetamol (assay 99.8\%) was collected from Beximco Pharmaceuticals Ltd., Dhaka. All materials used in the formulations were of pharma grade. Chemicals used for analytical and dissolution studies were of reagent grade.

Collection of commercial samples. Ten brands of paracetamol suspensions and one brand of paracetamol solution (syrup) were purchased from various drug shops of Dhaka city. The samples were checked for their production and expiry dates before purchasing. The suspensions were randomly coded from PM-1 to PM-10 and stored under appropriate conditions until further study. The labels of all the products claimed to contain $120 \mathrm{mg}$ of the active ingredient per $5 \mathrm{ml}$.

Dissolution studies. Dissolution of commercial suspensions was carried out using a paddle stirring assembly of USP XXII apparatus 2. Although mainly designed for tablets and capsules, this apparatus has also been used by several investigators to study the dissolution behaviour of suspensions. ${ }^{2,15,16} 500 \mathrm{ml}$ of $0.1 \mathrm{~N} \mathrm{HCl}$ was placed in the dissolution vessel which was allowed to equilibrate at a temperature of $37 \pm 1^{\circ} \mathrm{C}$. $10 \mathrm{ml}$ suspension was introduced carefully into the bottom of the apparatus. The paddle rotation was controlled at $25 \mathrm{rpm} .3 \mathrm{ml}$ of the sample was withdrawn with a glass syringes from a fixed position of the vessel at 3 minutes intervals and $3 \mathrm{ml}$ of fresh $0.1 \mathrm{~N} \mathrm{HCl}$ solution was added immediately as replacement. The collected samples were filtered and, after proper treatment, the amounts of paracetamol dissolved at 3 minutes intervals were determined by taking the absorbance at $430 \mathrm{~nm}$ following the same procedure as mentioned in the paracetamol quantification.

Viscosity measurement. The kinematic viscosity of the suspensions was measured using 'Cannon-Fenske-Routine' viscometer. For comparative purpose, a low-stress single point determination (shear rate 100 second $^{-1}$ ) was done and the readings were corrected and expressed in units of centistokes (cS).

Paracetamol quantification. To verify label claims, the paracetamol content of the commercial suspensions was estimated by a colorimetric method. ${ }^{17}$ This method is a minor modification of the method described in USP XXII (1989) for determination of paracetamol in paracetamol oral suspensions. ${ }^{18}$ Absorbance of the treated samples were measured at $430 \mathrm{~nm}$ against a blank and concentrations of paracetamol were determined using a properly constructed calibration curve.

Preparations of laboratory samples. Dispersions of the suspending agents were made and kept overnight to allow proper swelling. Propyl paraben $(0.03 \% \mathrm{w} / \mathrm{v})$ was used as preservative in all cases. Paracetamol suspensions were prepared (120 mg / $5 \mathrm{ml}, 2.4 \% \mathrm{w} / \mathrm{v}$ ) using mortar and pestle following standard procedures. ${ }^{4,5,15}$ All together, twenty five formulations were made but only those forming somewhat satisfactory suspensions and having significant findings are listed in Table 1 and discussed later.

\section{RESULTS AND DISCUSSION}

Various investigators have studied the dissolution behaviors of commercial samples of several 
drugs like nitrofurantoin, steroids, sulfonamides, antiinfectives etc. ${ }^{1,3,10}$ We selected ten locally available commercial samples of paracetamol suspensions (PM-1 to PM-10) for this study and all of them contained the active ingredient within the USP specified limits (within $\pm 10 \%$ of the label claim). Thus, all samples were found suitable for dissolution studies considering the label claim as $100 \%$ as recommended by the USP. We also investigated the dissolution behaviors of four (PL-1 to PL-4) laboratory-made samples of paracetamol suspensions. The suspensions contained different concentrations of the two widely used suspending agents, sodium carboxymethylcellulose 10,000 cps (Na-CMC) and microcrystalline cellulose (Avicel RC 591) in combination as shown in Table 1.

Table 1. List of laboratory made paracetamol suspensions

\begin{tabular}{ccc}
\hline $\begin{array}{c}\text { Formulation } \\
\text { code }\end{array}$ & \multicolumn{2}{c}{$\begin{array}{c}\text { Suspending agents (\%w/v of the } \\
\text { suspension)* }\end{array}$} \\
& CMC-Na 10000 cps & Avicel RC 591 \\
\hline PL-1 & 0.3 & 0.3 \\
PL-2 & 0.4 & 0.4 \\
PL-3 & 0.4 & 0.5 \\
PL-4 & 0.5 & 0.4 \\
\hline
\end{tabular}

Treatment of dissolution data. The dissolution data for each commercial sample (one syrup and ten suspensions) and laboratory made suspensions were treated by converting observed drug concentration at each sampling time (3 minutes interval) and, in turn, to percents dissolved based on the labeled claim as $100 \%$. Analysis of dissolution data demonstrated a marked difference in the dissolution characteristics of different samples (Figures 1a \& 1b). There is no official specification for the minimum limit of dissolution of paracetamol suspensions within a specified period of time. However, the USP specifies a value of not less than $80 \%(\mathrm{Q})$ within 30 minutes for paracetamol tablets. ${ }^{18}$ In this context, the dissolution of the suspensions were found to be much faster as expected since almost 100\% drug was dissolved from all the products (except PM-10) within 30 minutes even at slow stirring speed of 25 rpm. However, considering paracetamol release at earlier time intervals, it is evident that differences exist among the test products regarding the rate of drug dissolution. For instance, extent of drug release varied between $72 \%$ and $100 \%$ at 15 minutes interval among the products exempting PM-10.

*Each formulation contains $2.4 \% \mathrm{w} / \mathrm{v}$ paracetamol and $0.03 \%$ propylparaben.

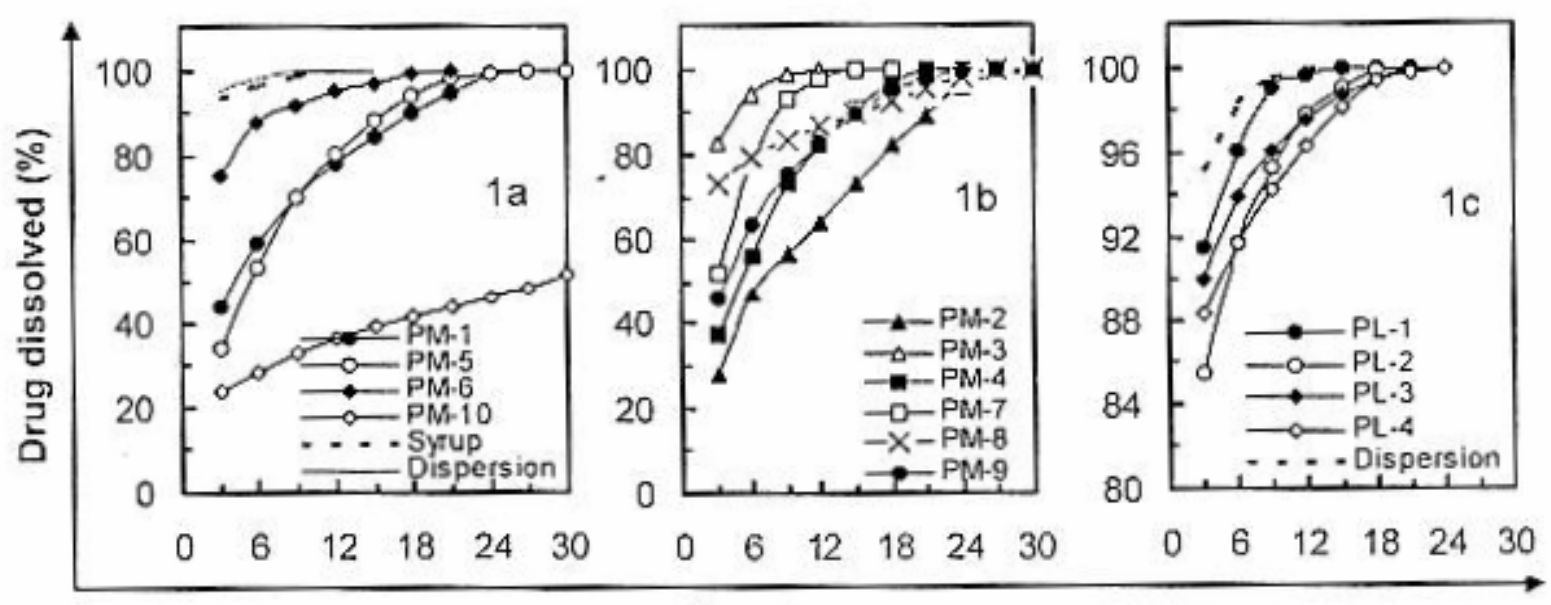

Time (minutes)

Figure 1. Dissolution profiles of commercial (1a and 1b) and laboratory-made (1c) paracetamol suspensions

The Paracetamol syrup (marketed sample) and aqueous dispersion of Paracetamol (paracetamol powder dispersed in water alone) were found almost similar in dissolution behavior. The sample PM-3 showed a very high rate of in vitro dissolution similar to that of the syrup. Thus, this product is expected to 
give a very quick onset of action. The brands PM-1, PM-4, PM-5, PM-6, PM-7, PM-8 and PM-9 showed moderate drug release pattern; more than $80 \%$ of the drug was dissolved within 15 minutes. In case of brand PM-2, the initial drug release pattern was slow and after 15 minutes, about $70 \%$ of the drug was dissolved. In case of PM-10 paracetamol dissolution was found to be $39 \%$ and $52 \%$ at 15 minutes and 30 minutes intervals respectively indicating a much reduced rate and extent of drug release. These findings suggests that there may be differences in the onset of action among the commercial products and at least one of them (PM-10) is likely to exhibit serious bioavailability problems. However, these can only be confirmed performing suitable in vivo testing.

As mentioned earlier, polymers used in suspensions have been found to interfere with the dissolution of drugs from these dosage forms. ${ }^{3-8}$ The polymers, used as suspending agents, are essential components of suspension formulations and need to be carefully selected. In order to access the affect of suspending agents on paracetamol dissolution, we formulated several paracetamol suspensions in our laboratory using different concentrations and combinations of widely used suspending agents. Formulations of four such suspensions (Table 1) showing better physical properties in terms of sedimentation volume, redispersibility, viscosity and elegancy were subjected to dissolution studies following the same procedures as mentioned for commercial products. The dissolution profiles of the laboratory made suspensions (PL-1 to PL-4) are shown in Figure 1c. It is clearly evident that the suspensions containing CMC-Na and Avicel as suspending agents showed retarded dissolution than that observed for simple dispersion of' paracctamol powder in water containing no suspending agent. Increase in the concentration of suspending agents decreased the dissolution rate as expected although the final extent of drug dissolution was almost $100 \%$. As depicted from Figure 1c, the retarding effect of CMC-Na seems to be a little more pronounced than that of Avicel. Investigators have suggested that retardation of' drug dissolution could be due to entrapment of the drug in the polymer chain or adsorption of' polymer on the drug particles. This would prevent easy asses of' the solvent to the drug particles. ${ }^{6}$ There are some reports in which drug dissolution was somewhat enhanced at very low concentrations of hydrophilic suspending agents in presence of surfactants like polysorbate 80 (Tween 80 ). ${ }^{15}$ Others have studied the influence of particle size distribution, zeta potential, flocculation extent, complexation etc. which will subsequently affect drug dissolution. ${ }^{3,15}$

Viscosity considerations. The present study also revealed that dissolution rate was related to the viscosity of suspensions, either in commercial products or in laboratory made samples. In general, low viscosity samples showed faster dissolution rates and vice versa. In the laboratory made samples, viscosity increased with increase of concentration of' suspending agents, which, in turn, decreased the dissolution rate. Viscosity showed a strong correlation with the extent of dissolution at the initial stage (3 minutes) but, with the lapse of time, the relationship became gradually weaker as observed from the decreasing values of correlation coefficient at later time intervals (6 minutes, 9 minutes, 12 minutes and 15 minutes) (Figure 2).

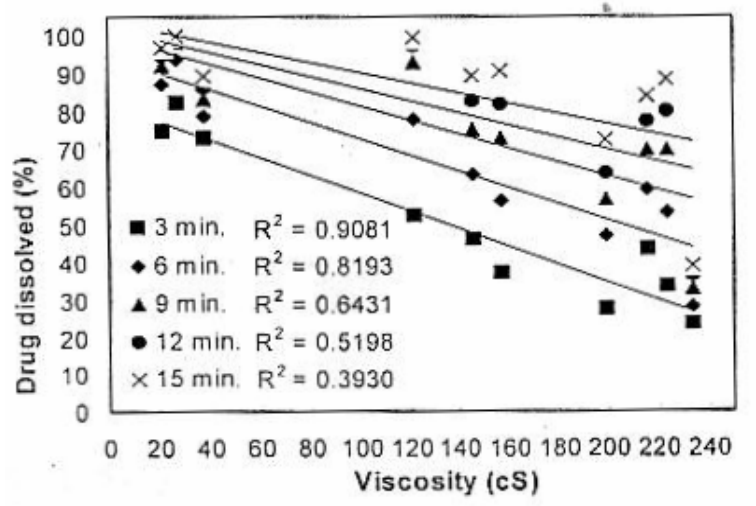

Figure 2. Correlation between viscosity and drug dissolved at different time intervals for commercial paracetamol suspensions

It has been postulated that their is a possibility of formation of regions of' high viscosity, both in the tightly bound layer surrounding the drug particles as well as in the bulk dissolution medium due to 
the hydrated polymer chains causing resistance in the diffusion process. ${ }^{6}$ Various other workers have also confirmed the retarding effect of' viscosity on diffusion, gastrointestinal transit and absorption of drugs. ${ }^{19,20}$ However, other investigators have showed that viscosity may have very little or no effect on drug dissolution if the volume of the dissolution medium is very large or if other factors are dominant. ${ }^{3}$

Kinetic analysis. The dissolution rate was subjected to various mathematical models like zero order, first order, Higuchi and Hixson-Crowell equations to elucidate the kinetic behavior of drug release from the test samples. It was observed from the correlation coefficient values that the release kinetics of' paracetamol from the suspensions appeared to follow first order release kinetics (log of percent drug remaining versus time) in most of tile cases of' both commercial and laboratory-made paracetamol suspensions (Table 2). However, Higuchi model (percent drug dissolved versus square root of' time) was also in good agreement in many cases. Thus, complex mechanisms, influenced by various factors, are likely to be involved in the release of drugs from suspension dosage forms. ${ }^{3,4,15}$

Table 2. Correlation coefficient values $\left(\mathrm{r}^{2}\right)$ obtained from the two most obeyed kinetic models for commercial and laboratory-made suspension

\begin{tabular}{ccc}
\hline \multirow{2}{*}{ Sample code } & \multicolumn{2}{c}{ Correlation coefficients $\left(\mathrm{r}^{2}\right)$} \\
\cline { 2 - 3 } & First order equation & Higuchi equation \\
\hline PM-1 & 0.9796 & 0.9848 \\
PM-2 & 0.9083 & 0.9902 \\
PM-3 & 0.9945 & 0.8494 \\
PM-4 & 0.9716 & 0.9598 \\
PM-5 & 0.9556 & 0.9188 \\
PM-6 & 0.9685 & 0.9151 \\
PM-7 & 0.9680 & 0.8635 \\
PM-8 & 0.9299 & 0.9955 \\
PM-9 & 0.9632 & 0.9259 \\
PM-10 & 0.9782 & 0.9978 \\
PL-1 & 0.9897 & 0.8474 \\
PL-2 & 0.9619 & 0.9432 \\
PL-3 & 0.9201 & 0.9382 \\
PL-4 & 0.9587 & 0.9673 \\
\hline
\end{tabular}

\section{CONCLUSION}

Quick onset of action is desired in case of immediate release suspensions. Pharmaceutical manufactures should give due attention to dissolution characteristics of suspensions similar to those given for solid and semisolid dosage forms. Possible drug and excipients interaction should be considered during selection of suspending agents added to increase viscosity and retard sedimentation. Although various devices have been utilized, the rotating paddle method using an aqueous dissolution medium is recommended medium method for dissolution testing of suspensions. ${ }^{21}$ The in vitro stirring rate (25 rpm or even low) should be selected to facilitate discrimination among batches with different release properties. $^{21}$ Such low stirring is also a more reasonable simulation of the mild in vivo gastric agitation. In conclusion, the importance of dissolution testing for suspensions and proper selection of the suspending agents during their formulations should be dully emphasized to ensure batch-to-batch reproducibility and consistent pharmacological/biological activity.

\section{REFERENCES}

1. Mathur, L.K., Jaffe, J.M., Poust, R.I., Harry, H., Goehl, T.J., Shah, V.P. and Colaizzi, J.L. 1979. Bioavailalivity and dissolution behaviour of trisulfapyrimidine suspensions. $J$. Pharm. Sci. 68, 699-701.

2. Strum, J.D., Colaizzi, J.L., Goehl, T.J., Jaffe, J.M., Pitlick, W.H., Shah, V.P. and Poust, R.I. 1978. Bioavailalivity of sulfonamide suspensions I: Dissolution profiles of sufamethizole using paddle method. J. Pham. Sci. 67, 1399-1402.

3. Howard, S.A., Mauger, J., Hsieh J.W. and Amin, K. 1979. Suspending agent effects on steroid suspension dissolution profiles. J. Pham. Sci. 68, 1475-1479.

4. Barzegar-Jalalim, M. and Richardsm, J.H. 1979. The effect of suspending agents on the release of aspirin from aqueous suspensions in vitro. Int. J. Pharmaceutics. 2, 195-201.

5. Hashem, F. and El-Said, Y. 1987. Effect of suspending agents on the characteristics of some anti-inflammatory suspensions. Pharmazie. 42, 732-735.

6. Shah, N.B. and Sheth, B.B. 1976. Effect of polymers on dissolution from drug suspensions. J. Pharm. Sci. 65, 16181623. 
7. Reddy, R.K., Khalil, S.A. and Gouda, M.W. 1976. Effect of dioctyl sodium sulfosuccinate and poloxamer 188 on dissolution and intestinal absorption of sulfadiazine and sulfisoxazole in rats. J. Pharm. Sci, 65, 115-118.

8. 'I'ermpio, J.S. and Zatz, J.L. 1980. Flocculation effect of xanthan gum in pharmaceutical suspensions. J. Pharrn. Sci, 69, 1209-1214.

9. Zatz, J.L. 1979. Effect of formulation additives on flocculation of dispersions stabilized by a nonionic surfactant. Inl. J. Pharmaceuticals. 4, 83-86.

10. Bates, T.R., Rosenberg, H. and Tembo, A. 1973. Inconsistencies in rationale underlying official USP dissolution rate specifications for nitrofurantoin. $J$. Pharm. Sci. 62, 2057-2058.

11. Bates, T.R., Lambert, D.A. and Johns, W.H. 1969. Correlation between the rate of' dissolution and absorption of salicylamide from tablet and suspension dosage. $J$. Pharm. Sci. 58, 1468-1470.

12. Seager, H. 1968. The effect of melhycellulose on the absorption of nitrofurantoin from the gastrointestinal tract. J. Pharm. Pharmacol. 20, 968-969.

13. Berlin, A., Simers, B., Agurell, S., Hiort, A., Sjoright, F. and Storm, S. 1972. Determination of bioavailability of diazepam in various formulations from steady state plasma concentration data. Clin. Pharmcol. Ther. 19, 733-743.
14. Shahidi, R.U. 2006. Quick Index Medicinal Products and Problems-13. Dhaka. pp. 424-426.Hewitt, R.R. and Levy, G. 1971. ibid, 60, 784. 1971.

15. Venkateshwarlu, G., Rambu, D., Ramesh, B.R. and Sarveswar Rao, V.V. 1990. Dissolution kinetics of rifampicin aqueous suspension. Indian Drugs. 28, 8-12.

16. Abdou, HM. 1995. In: Remington's Pharmaceutical Sciences (Gennaro, A.R. Ed.). Mack Publishing Company, Pennsylvania. 17th edilion, Chapter 35. p.604.

17. Sethi, P.D. 1997. In: Quantitative Analysis of Drugs in Pharmaceutical Dosage Forms, CBS Publishers and Distributor, New Delhi. $3^{\text {rd }}$ edition, pp. 143-145.

18. USP XXII, 1990, United States Pharmacopeial Convention Inc., Rockvilli, MD. p.14.

19. Hewitt, R.R. and Levy, G. 1971. Effect of viscosity on thiamine and riboflavin absorption in man. J. Pharm. Sci. 60, 784-786.

20. Ashley, J.J. and Levy, G. 1973. Effect of viscosity and an antocholinergic agent on the bioavailability of a poorly absorbed drug (phenolsulfonphthalein) in man. J. Pharm. Sci. 62, 688-690.

21. Siewert, M., Dreeman, J., Brown, C.K. and Shah, V.P. 2003. FIP/AAPS Guidelines to dissolution/in vitro release testing of novel/special dosage forms. AAPS Pharm. Sci. Tech. 4, article 7 (web: http://aapspharmscitech.org) 